\title{
DERIVATION OF PRESSURE GRADIENTS FROM MAGNETIC RESONANCE ANGIOGRAPHY USING MULTI-RESOLUTION SEGMENTATION
}

\author{
P E Summers, A H Bhalerao \\ United Kingdom
}

The use of magnetic resonance (MR) angiography in cardiovascular assessment is steadily increasing on the merits of sensitivity to disease states, lack of ionising radiation and iodinated contrast agent, and widespread applicability to the vasculature. Because MR angiograms are usually obtained as a data set of parallel images, visualisation and analysis require post-processing. The sparsity of the macro-vasculature relative to the data volume, coupled with the still variable results of MR studies has led to general acceptance of very few widely used processing and viewing tools in clinical practice.

We have implemented a model based multiresolution, image segmentation technique which makes use of the velocity field information in phase contrast MR angiograms, and an a priori assumption that locally, blood vessels appear as line segments. The list structure of the resulting segmentation can be used efficiently in subsequent image analysis and processing. Within this segmentation, flow direction, vessel axis, diameter and velocity estimates can be made. We demonstrate its use in extracting vessels from patient data and in the calculation of pressure gradients in a model stenosis.

\section{BACKGROUND}

Phase contrast magnetic resonance (MR) angiography establishes a linear relationship between a component of velocity and signal phase which is mapped in an image in the same way as the signal intensity, van Dijk (1). It is possible then, to produce a three dimensional field map of orthogonal velocity components and a value of the signal intensity. The velocity components may be combined by the square root of the sum of squares to produce a speed image. In the speed images, vessels are typically displayed as bright against a background of static tissue. It is difficult, however, to collate planar images mentally to form a unified view of the vascular tree. Image processing and rendering techniques are therefore used to visualise the distribution of blood vessels. The simplest, and most widely used of these is the maximum intensity projection (MIP). Here, a projection through the data set is formed with the brightest value along each path through the data being represented in the resultant images. Surface rendering of MR angiographic data is also possible, but requires the selection of a segmentation threshold. Such approaches are limited to qualitative assessment of the anatomy, Cline et al (2).

Clinically, the end points of an angiographic study are a series of requests. The coarsest assessment is strictly anatomic: is there a patent vessel present? In complicated anatomy (such as the circle of Willis), the direction of flow is the next level of description. More commonly, the calibre of the vessel is of interest. This provides a measure of the extent of arterial disease, and the amount of work done in carrying blood past a given point. Further to this, pressure and shear in the flow, and strain and compliance in the vessel wall are measures of interest in understanding the progression of vascular disorders.

The velocity information contained in phase contrast angiograms can be used to assess higher clinical questions. Flow direction and velocity can be evaluated based on the velocity component images. The streamlines of flow can be estimated by following the velocity estimates for a collection of origin points, Buonocore (3), and De Becker (4).

The velocity $\mathbf{v}$ and pressure $\mathrm{p}$ fields are related by the Navier-Stokes equations:

$\nabla \mathrm{p}=\mu \nabla^{2} \mathbf{v}-\rho(\partial \mathbf{v} / \partial \mathrm{t}+\mathbf{v} \cdot \nabla \mathbf{v}-\mathbf{g})$

where $\mu$ and $\rho$ are the fluid viscosity and density respectively, and $\mathbf{g}$ represents body forces such as gravity on the fluid. A further condition $\nabla \cdot \mathbf{v}=0$ can be included for an incompressible fluid. Sufficient knowledge of the velocity field allows the pressure gradient field to be calculated. There is a degree of complementarity in having such a dual representation of the vasculature. The velocity profile is naturally related to the shear at the vessel wall, while the pressure is associated with the vessel distension, Chorin (5). The distinction is important in some clinical settings. The shear relates to the likelihood of plaque fragments dissociating from the vessel wall with the risk of emboli forming downstream. Pressure is more closely related to the risk of rupture of a vessel in conditions such as aneurysm, Hademenos (6).

Interrogating haemodynamics is complicated by the fact that the majority of the data is from air or static tissue. The lack of signal from air can be used as a mask to zero the velocity estimates for these regions. These zero values however, remain part of the volume of data which must be processed. To avoid processing data from nonvascular regions, intermediate processing is needed. Other authors have demonstrated segmentation of MR angiograms based on speed or intensity images weighted by the speed of flow, Szeleky et al (7). 
We have adopted a multi-resolution image model where the centre lines of blood vessels are modelled as interconnected, piece-wise linear, 3D curves. The model parameters, and hence the segmentation, are obtained by first expanding the image data into an oct-tree representation. Curve segments representing the current best fit of the data to the model are identified across resolutions. Probable curves are then inferred through these 'trace points' by means of an adjacency graph, forming the basis of a symbolic description, Bhalerao (8), Calway and Wilson (9). Isolation of regions believed not to contain vessels at a higher level, and their exclusion from processing at a lower levels can make subsequent processing more efficient than if applied directly on the high resolution data.

\section{METHODS}

The multi-resolution segmentation is based on an octtree averaging on the combined set of velocity component images. At successive scales, a level of confidence test was made to estimate the occupancy probability of the blocks. Blocks satisfying the coherence criteria were further assessed to form the segmentation. Orientation of flow within each block was based on the direction of maximum coherence among the velocity components. The offset of the flow feature in each block was estimated using a Hough transform over the daughter voxels. End-points of the flow feature, an estimate of the feature's cross-sectional area (obtained by least squares fitting), the block reference (relative to parent and child blocks), were combined with the velocity orientation and magnitude calculated for each block.

Phase contrast angiograms obtained as part of an ongoing clinical trial were subjected to the segmentation processing. Coronal images of the cranial vessels with a total of 64 slices, each $1.0 \mathrm{~mm}$ thick were acquired. A velocity sensitive range of $50 \mathrm{~cm} / \mathrm{s}$ was used to minimise artefacts in the feeding arteries.

Under steady flow, a phase contrast angiogram of 140 cross-sectional, $0.8 \mathrm{~mm}$ thick images of a $19.1 \mathrm{~mm}$ bore tube under steady flow with a $75 \%$ area stenosis were acquired. This was used as a test data set for calculation of the pressure gradient calculations. The velocity encoding range was $50 \mathrm{~cm} / \mathrm{s}$ in three orthogonal directions. A small deviation between the tube axis and imaging planes was seen.

Following segmentation, data from the inlet to the stenosis of the tube phantom was subjected to a modified Navier-Stokes equation to estimate the pressure gradient as a function of position in the tube. The system was assumed to be time independent, and to lie in a horizontal plane such that the time derivative $(\mathrm{dv} / \mathrm{dt})$ and body forces $(\rho \mathbf{g})$ could be ignored. The axial component of the pressure gradient was studied using a discretised version of the Navier-Stokes equation:

$$
\begin{array}{r}
\partial \mathrm{p} / \partial \mathrm{z}=\mu\left(\partial^{2} \mathrm{v}_{\mathrm{z}} / \partial \mathrm{x}^{2}+\partial^{2} \mathrm{v}_{\mathrm{z}} / \partial \mathrm{y}^{2}+\partial^{2} \mathrm{v}_{\mathrm{z}} / \partial \mathrm{z}^{2}\right) \\
-\rho \mathrm{v}_{\mathrm{z}}\left(\partial \mathrm{v}_{\mathrm{z}} / \partial \mathrm{x}+\partial \mathrm{v}_{\mathrm{z}} / \partial \mathrm{y}+\partial \mathrm{v}_{\mathrm{z}} / \partial \mathrm{z}\right)
\end{array}
$$

Each feature block identified in the segmentation was included in the calculation of local pressure gradient. The cross-sectional average estimates of velocity, area and pressure gradient were calculated. Comparison measurements of cross-sectional area and mean velocity based on scanner software were also made.

\section{RESULTS}

The stages in the segmentation process are shown for the in-vivo study of the circle of Willis in Figure 1. Having chosen a threshold which minimises the number of disjoint structures in the reconstruction, the resulting surface rendering of the speed images is seen in Figure 1a. The blocks determined by the single linear vessel feature are shown in Figure 1b. From each block, the velocity and vessel cross-sectional area estimates are shown in Figure 1c and d respectively.

Figure 2a shows a MIP of the phase contrast images of the inlet to the tube stenosis. The narrowing of the tube is well seen. The flow feature estimates for the inlet are shown in Figure 2b. The feature threshold was deliberately set low to allow changes in the velocity field to be demonstrated. Figure $2 \mathrm{c}$ demonstrates the shear contribution to the pressure gradient along the axis of the tube. Profiles of the cross-sectional mean diameter, velocity and axial component of the pressure gradient along the tube in the inlet region are shown in Figure 2d. This illustrates the increase in velocity at the stenosis, the reduction in cross-section and changes in pressure gradient. At the Reynolds numbers of this study, turbulence would not be expected, Chorin (5).

From a patient data set of $64^{3}$ voxels the segmentation produced a point list of 466 values. A point list of 167 elements was obtained from a similarly sized data set for the tube phantom. Processing time for the segmentation and calculation of the velocity gradient required less than one minute on a standard unix workstation.

\section{DISCUSSION}

By using the velocity component images from phase contrast angiograms we have been able to estimate a number of parameters including flow velocity, direction and pressure gradient from a single imaging study. The coherence criteria in segmentation and the pressure gradient calculation are both subject to constraints on image resolution, and coherence of the flow, Strang et al (10). Application of the segmentation method to in-vivo vasculature does not appreciably degrade the data in cases we have studied. The sensitivity of the pressure 
gradient calculation to noise through derivatives of the signal can be expected to be increase in the less controlled setting of in-vivo imaging.

One of the factors not taken into our implementation was temporal variations in pressure. By acquiring timeresolved images, the time dependent term can be incorporated in the haemodynamic equations. Other authors have studied pressure gradients across single slice images as a function time during the cardiac cycle while neglecting the significant spatial variation terms Urchuk (11), Eichenberger (12). Currently however, phase contrast angiography techniques on standard MR scanners are too slow for in-vivo studies which are both volumetric and time-resolved. Faster acquisition techniques would allow the full study of spatial and temporal pressure gradient behaviour, Song (13), and Pike (14).

\section{CONCLUSIONS}

There is an increasing need for efficient and effective visualisation tools in medical imaging. Making use of as much information as possible early in the processing can greatly speed subsequent calculations. A multiresolution, model based approach to the segmentation of phase contrast angiograms allows extraction of a number of parameters which are clinically relevant. An extension to the segmentation used here is the subject of ongoing work to incorporate branching points in the model.

The 3D spatial analysis presented determines the pressure field over an extended region of the vasculature. Extension to a four dimensional case will require inclusion of the time derivative term in the Navier-Stokes equation. The segmentation process can be fed into other analyses and visualisation tools such as anisotropic maximum intensity projections to reduce background intensity, and potentially to provide vessel surface estimates for surface rendering, or streamline estimation.

\section{REFERENCES}

1. van Dijk P, 1984, "Direct cardiac NMR imaging of heart wall and blood flow velocity", JCAT, 8(3), 429435

2. Cline H, Dumoulin C, Lorensen W, Souza S, Adams W, 1991, "Volume rendering and connectivity algorithms for MR angiography", MR Med, 18, 384394

3. Buonocore M, 1994, "Algorithms for improving calculated streamlines in 3D phase contrast angiography", MR Med, 31, 22-30

4. De Becker J, Fuderer M, Kouwenhoven M, 1994, “3D Flow visualisation in phase contrast angiography", Proc of SMR, San Francisco, USA, 450
5. Chorin A, Marsden J, 1990, “A mathematical introduction to fluid mechanics", Springer Verlag, New York, USA

6. Hademenos G, 1995, "The Physics of Cerebral Aneurysms", Physics Today, 48 (2), 24-30

7. Szeleky G, et al, 1994, "Structural description and combined 3-D display for superior analysis of cerebral vascularity from MRA", Proc. Visualisation in Biomed Comp, Rochester, USA, 272-281

8. Bhalerao A, Summers P, 1995, “A multiresolution approach to flow feature extraction from phase contrast MRA data", Proc. IEE Colloquium on Multires modelling and Analysis in Image Proc and Comp Vision, in press

9. Calway A, Wilson R, 1990, "Curve extraction in images using the multiresolution Fourier transform", Proc IEEE Conf Acoust, Speech and Signal Proc, 21292132

10. Strang J, Herfkens R, Pelc N, 1994, "Voxel size effects on vascular shear measurement", Proc SMR, San Francisco, 1002

11. Urchuk S, Plewes D, 1994, "MR measurements of pulsatile pressure gradients”, JMRI, 4, 829-836

12. Eichenberger A, Jenni R, von Schulthess G, 1992, "Aortic valve pressure gradients in patients with aortic valve stenosis: Quantification with velocity encoded cine MR imaging", AJR, 160, 971-977

13.Song S, Leahy R, Boyd D, Brundge B, 1994, "Determining cardiac velocity fields andintraventricular pressure distribution from a sequence of ultrafast CT images", IEEE Trans Med Imaging, 13 (2), 386-397

14. Pike B, Meyer C, Brosnan T, Pelc N, 1994, "Magnetic resonance velocity imaging using a fast spiral phase contrast sequence", MR Med, 32, 476-483 